\title{
Revisiting successful transplantation with marginal lungs: Fourteen years later, a new era of extended criteria
}

\author{
J. Hunter Mehaffey, MD, Robert B. Hawkins, MD, Eric J. Charles, MD, and Curtis G. Tribble, MD, \\ Charlottesville, Va
}

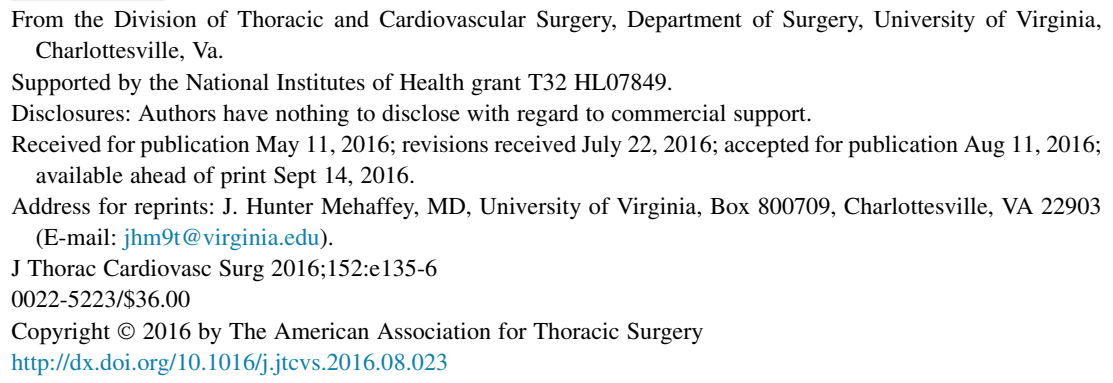

There continues to be a shortage of donor lungs, despite years of research into improving marginal lungs before transplantation. We revisit a case reported in 2001 in which marginal lungs were successfully transplanted with the help of extracorporeal membrane oxygenation (ECMO) in the immediate postoperative period. ${ }^{1}$ Although ex vivo lung perfusion (EVLP) holds promise for improving marginal lung utilization, this case highlights an alternative approach in which lungs are allowed to rehabilitate in vivo.

\section{CLINICAL SUMMARY}

The recipient was a 55-year-old woman with end-stage respiratory failure from pulmonary fibrosis. Her pulmonary function was poor, with a forced expiratory volume in $1 \mathrm{sec}-$ ond of $0.71 \mathrm{~L}$ ( $33 \%$ predicted), a forced vital capacity of $0.85 \mathrm{~L}$ ( $30 \%$ predicted), and a baseline oxygen requirement of $5 \mathrm{~L} / \mathrm{min}$. On the basis of these data, her survival without transplant was estimated to be 3 months. On notification of the availability of a suitable donor and arrival at the hospital for the expected transplant, the patient was profoundly dyspneic on $5 \mathrm{~L}$ of oxygen and thought to have had her respiratory failure further advanced. The donor lungs were from a young person with brain death who had abrupt and severe pulmonary edema develop after the decision was granted for organ donation. The ratio of $\mathrm{Po}_{2}$ to fraction of inspired oxygen at the time of procurement was 133, down from 414 earlier in the day. In light of worsening chest radiography, negative drug screen results, normal blood pressure, and absence of fluid administration, the origin of the pulmonary edema was thought to be neurogenic. The high-risk nature of the donor lungs was discussed in detail, including the virtual certainty of the need for ECMO after transplantation. Ultimately the decision was made to proceed with the transplant. ${ }^{1}$

After the uneventful implantation of both lungs, as anticipated the patient could not be separated from

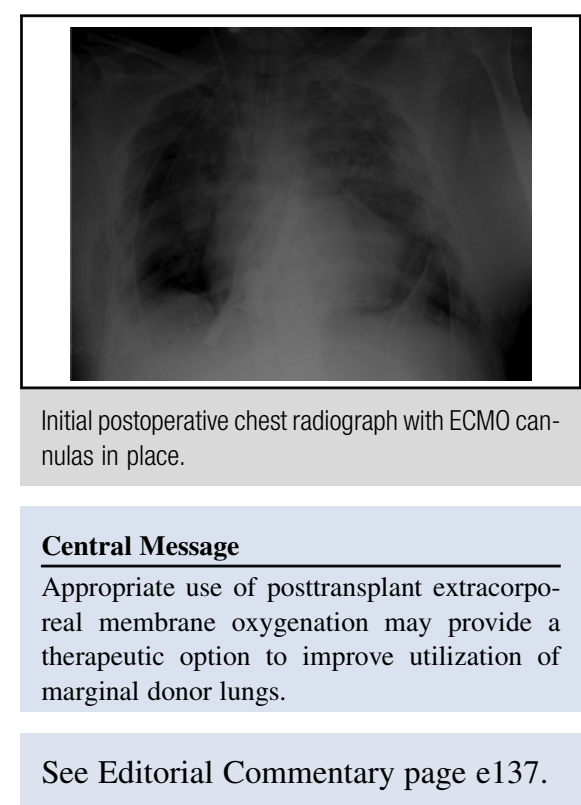

cardiopulmonary bypass because of persistent hypoxemia, and right atrium-pulmonary artery ECMO support was initiated as described previously. ${ }^{1}$ After 2 days, the patient was weaned from the ECMO circuit and decannulated. Early after transplant, she had excellent pulmonary function, with a forced expiratory volume in 1 second of $1.95 \mathrm{~L}(96 \%$ predicted) and a forced vital capacity of $2.37 \mathrm{~L}$ ( $87 \%$ predicted). She was followed up for 14 years and did exceedingly well during that time frame, remaining stable on a regimen of tacrolimus and prednisone with only 1 transplant-related hospital readmission. She led a normal life, going back to work full time and traveling around the world. Her husband reports that she played with her grandkids, gardened, took care of their home and yard, and had a load of hobbies. Her pulmonary function remained normal, with a forced expiratory volume in 1 second of $1.24 \mathrm{~L}(67 \%$ predicted) and a forced vital capacity of $1.79 \mathrm{~L}(73 \%$ normal) 14 years after transplant (Figure 1). Unfortunately, at age 69 , toward the end of her life, the patient had neurocognitive decline, became quite frail, and eventually died of non-transplant-related causes at home with her husband.

\section{DISCUSSION}

Despite severe neurogenic pulmonary edema in these donor lungs, we demonstrated good long-term lung 


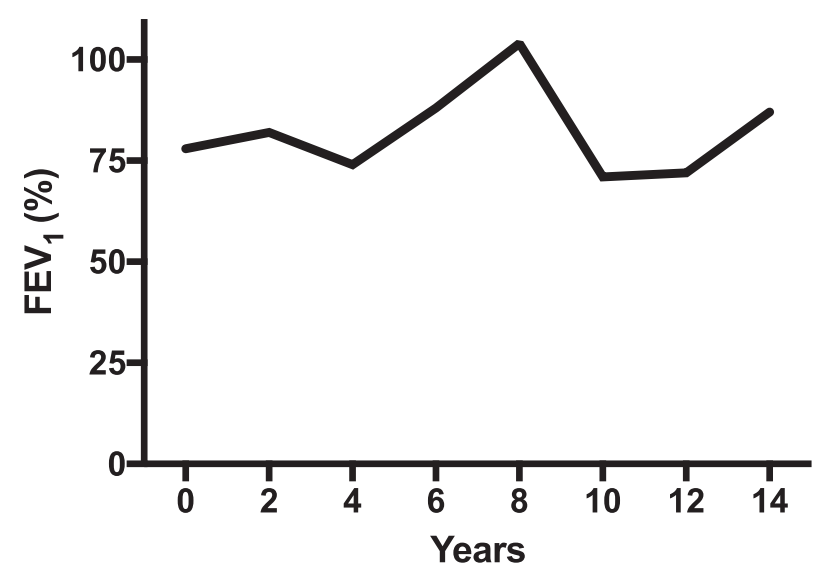

FIGURE 1. Patients yearly posttransplant forced expiratory volume in 1 second $\left(F E V_{1}\right)$.

function after a brief period of postoperative ECMO support. ${ }^{1}$ Considering the fact that lungs carry the worst transplant outcomes of all organs, transplant teams are hesitant to procure and transplant lungs without clear evidence of high quality. ${ }^{2}$ In addition, there are ethical issues to consider when obtaining recipient consent for marginal lungs that may require prolonged postoperative support and ECMO. Animal studies have shown, however, that marginal lungs can be successfully transplanted and that any subsequent ischemia-reperfusion injury is manageable. ${ }^{3-5}$ It is important to recognize different types of pulmonary edema with different presentations and clinical courses. Neurogenic pulmonary edema tends to have a rapid onset, leading to non-diffuse alveolar damage acute respiratory distress syndrome, unlike other causes such as pneumonia or aspiration, which cause acute respiratory distress syndrome with diffuse alveolar damage.

Increasing procurement rates and improving the utilization of marginal lungs may help alleviate the current donor lung shortage. EVLP is gaining clinical acceptance as a platform for lung assessment, rehabilitation, and prolonged transport before transplant. In this particular case, the postoperative use of ECMO provided support for the patient while allowing the lungs to recover in vivo under controlled conditions. This use of ECMO to support the transplant recipient and allow time for pulmonary function improvement may provide an additional alternative approach to increase marginal lung utilization. Currently, rates of primary graft dysfunction and chronic rejection continue to influence transplant teams to be conservative when evaluating donor lungs. With improved perioperative use of EVLP and ECMO, however, imperfect donor characteristics may not preclude procurement and transplant. Becoming comfortable with the idea of rehabilitating marginal donor lungs, with EVLP or ECMO as described here, may help maximize the number of lungs transplanted and decrease wait list mortality.

We give special thanks to Heidi Flanagan, RN, and Mark Robbins, MD, for their assistance with compiling data and updating the record. In addition, we thank the image management team at UVA for locating and converting a 20 -year-old chest radiograph.

\section{References}

1. Fiser SM, Kron IL, Long SM, Kaza AK, Robbins M, Kern JA, et al. Donor lung salvage after neurogenic pulmonary edema with the use of post-transplant extracorporeal membrane oxygenation. J Thorac Cardiovasc Surg. 2001;122:1257-8.

2. Ailawadi G, Lau CL, Smith PW, Swenson BR, Hennessy SA, Kuhn CJ, et al. Does reperfusion injury still cause significant mortality after lung transplantation? J Thorac Cardiovasc Surg. 2009;137:688-94.

3. Yeung JC, Cypel M, Waddell TK, van Raemdonck D, Keshavjee S. Update on donor assessment, resuscitation, and acceptance criteria, including novel techniques-non-heart-beating donor lung retrieval and ex vivo donor lung perfusion. Thorac Surg Clin. 2009;19:261-74.

4. Cypel M, Yeung JC, Hirayama S, Rubacha M, Fischer S, Anraku M, et al. Technique for prolonged normothermic ex vivo lung perfusion. J Heart Lung Transplant. 2008;27:1319-25.

5. Wagner CE, Pope NH, Charles EJ, Huerter ME, Sharma AK, Salmon MD, et al. Ex vivo lung perfusion with adenosine A2A receptor agonist allows prolonged cold preservation of lungs donated after cardiac death. J Thorac Cardiovasc Surg. 2016;151:538-45. 